\title{
Chronic kidney disease is associated with a risk of higher mortality following total knee arthroplasty in diabetic patients: a nationwide population-based study
}

\author{
Liang-Tseng Kuo ${ }^{1,2}$, , Su-Ju Lin ${ }^{3}$, Chi-Lung Chen ${ }^{1}$, Pei-An Yu ${ }^{1}$, Wei-Hsiu Hsu ${ }^{1,4, *}$ and \\ Tien-Hsing Chen ${ }^{5, *}$ \\ ${ }^{1}$ Division of Sports Medicine, Department of Orthopaedic Surgery, Chang Gung Memorial Hospital, Chiayi, Taiwan \\ ${ }^{2}$ Chang Gung University of Science and Technology, Chiayi, Taiwan \\ ${ }^{3}$ Division of Nephrology, Department of Medicine, Chang Gung Memorial Hospital, Chiayi, Taiwan \\ ${ }^{4}$ College of Medicine, Chang Gung University, Taoyuan, Taiwan \\ ${ }^{5}$ Division of Cardiology, Department of Medicine, Chang Gung Memorial Hospital, Keelung, Taiwan \\ * These authors have contributed equally to this work \\ Correspondence to: Wei-Hsiu Hsu, email: 7572@cgmh.org.tw \\ Tien-Hsing Chen, email: skyheart0826@gmail.com \\ Keywords: total knee arthroplasty; diabetes mellitus; chronic kidney disease; mortality; periprosthetic joint infection \\ Received: August 16, $2017 \quad$ Accepted: September 24, $2017 \quad$ Published: October 31, 2017 \\ Copyright: Kuo et al. This is an open-access article distributed under the terms of the Creative Commons Attribution License 3.0 \\ (CC BY 3.0), which permits unrestricted use, distribution, and reproduction in any medium, provided the original author and source \\ are credited.
}

\section{ABSTRACT}

Diabetes and chronic kidney disease (CKD) are associated with a higher rate of complications in patients undergoing total knee arthroplasty (TKA). The purpose of this study was to determine the effects of CKD and diabetes in patients after TKA. Diabetic patients who received unilateral primary TKA between January 2008 and December 2011 were enrolled. The follow-up period was more than 6 months. The primary outcome was a TKA-related infection and the secondary outcome was all-cause mortality. The study cohort included 13844 patients who were followed for a mean period of 2 years, of whom 1459 (10.5\%) had CKD. The patients with CKD were older than those without CKD (71.6 versus 70.3 years, $P<0.0001)$ and had higher rates of hypertension, gouty arthritis, ischemic heart disease, chronic pulmonary obstructive disease, pulmonary embolism and deep vein thrombosis (all $P<0.0001)$. After adjustment of comorbidities, the CKD group had a higher incidence of urinary tract infections (OR: 1.61, 95\% CI: 1.19-2.17). There were no significant differences in wound infections, pneumonia, pulmonary embolism or in-hospital death between the two groups. After adjustment of confounders, the CKD group had higher rates of myocardial infarction (HR: 2.06, 95\% CI: 1.26-3.39) and mortality (HR: 1.99, 95\% CI: 1.59-2.48). The risk of TKA-related infection during follow-up was comparable between the two groups (HR: 1.31, 95\% CI: 0.94-1.82). In conclusion, CKD is associated with increased risks of urinary tract infections, myocardial infarction and all-cause mortality after TKA. Surgeons should be aware of this when evaluating TKA patients with renal disease.

\section{INTRODUCTION}

Periprosthetic joint infection (PJI) is a serious problem following total knee arthroplasty (TKA) $[1,2]$. The associated risk factors include surgical, environmental and host factors [3]. Orthopaedic surgeons have made great efforts to improve surgical outcomes, however medical comorbidities such as diabetes mellitus (DM) and chronic kidney disease (CKD) are still common risk factors leading to PJI $[2,4,5]$. 
It has been reported that patients with DM have more perioperative complications after TKA compared with patients without DM $[6,7]$. In addition, CKD has an impact on a patient's immunity, resulting in poorer outcomes following orthopaedic procedures [5, 8-11]. Furthermore, patients with end-stage renal disease have been reported to have a higher incidence of complications including infections after TKA [12-14]. However, no comparative study has investigated the effect of renal disease on the incidence of PJIs and associated outcomes following TKA in patients with DM.

The objectives of this study were to compare the incidence of complications and mortality following TKA in diabetic patients with and without nephropathy. We hypothesized that diabetic patients with nephropathy would have higher rates of complications such as PJI and poor surgical outcomes than those without nephropathy.

\section{RESULTS}

\section{Patient's characteristics}

Of the 13844 diabetic patients with primary TKA, 1459 had CKD (CKD group) and the remaining 12385 patents did not (non-CKD group) (Figure 1). As shown in Table 1 , the CKD group was 1.3 years older than the non-CKD group, and the CKD group had higher rates of hypertension, gouty arthritis, cirrhosis, ischemic heart disease, chronic obstructive pulmonary disease, malignancy, pulmonary embolism and a history of deep vein thrombosis (all $P<0.05$ ) (Table 1).

\section{In-hospital complications}

As shown in Table 2, the CKD group had a higher risk of developing urinary tract infections during the index admission than the non-CKD group (odds ratio [OR], 1.61 $95 \%$ CI $1.19-2.17, P=0.0020)$. There were no significant differences in other complications including pneumonia, acute PJI, or in-hospital death between the two groups.

\section{Outcomes after discharge of the index admission}

Table 3 shows the results of late complications including infection outcomes, cardiovascular events, readmission and all-cause mortality. With regards to infection outcomes, the CKD group had a trend of being prone to developing PJIs compared to the non-CKD group (hazard ratio [HR]: $1.31 ; 95 \% \mathrm{CI}, 0.94-1.82 ; P=0.1133$ ) after adjusting for possible confounding variables. With regards to cardiovascular events, the CKD group had a higher risk of acute myocardial infarction and major adverse cardiac events than the non-CKD group $(P<0.05)$. The cumulative incidence of PJI was slighter higher in the CKD group, although the difference was not statistically significant ( $P=0.0748$, competing risk survival model) (Figure 2).

The CKD group had a higher re-admission rate due to any cause at both 3 months of follow-up (HR, 1.52; 95\% CI, 1.33-1.75, $P<0.0001)$ and at the end of follow-up (HR, 1.39; 95\% CI, 1.28-1.50, $P<0.0001$ ). Compared to the non-CKD group, the CKD group also had a higher risk of mortality both at 3 months of follow-up (HR, 2.55; 95\% CI, 1.35-4.80, $P=0.0038$ ) and at the last follow-up (HR, $1.99 ; 95 \%$ CI, 1.59-2.48, $P<0.0001)$.

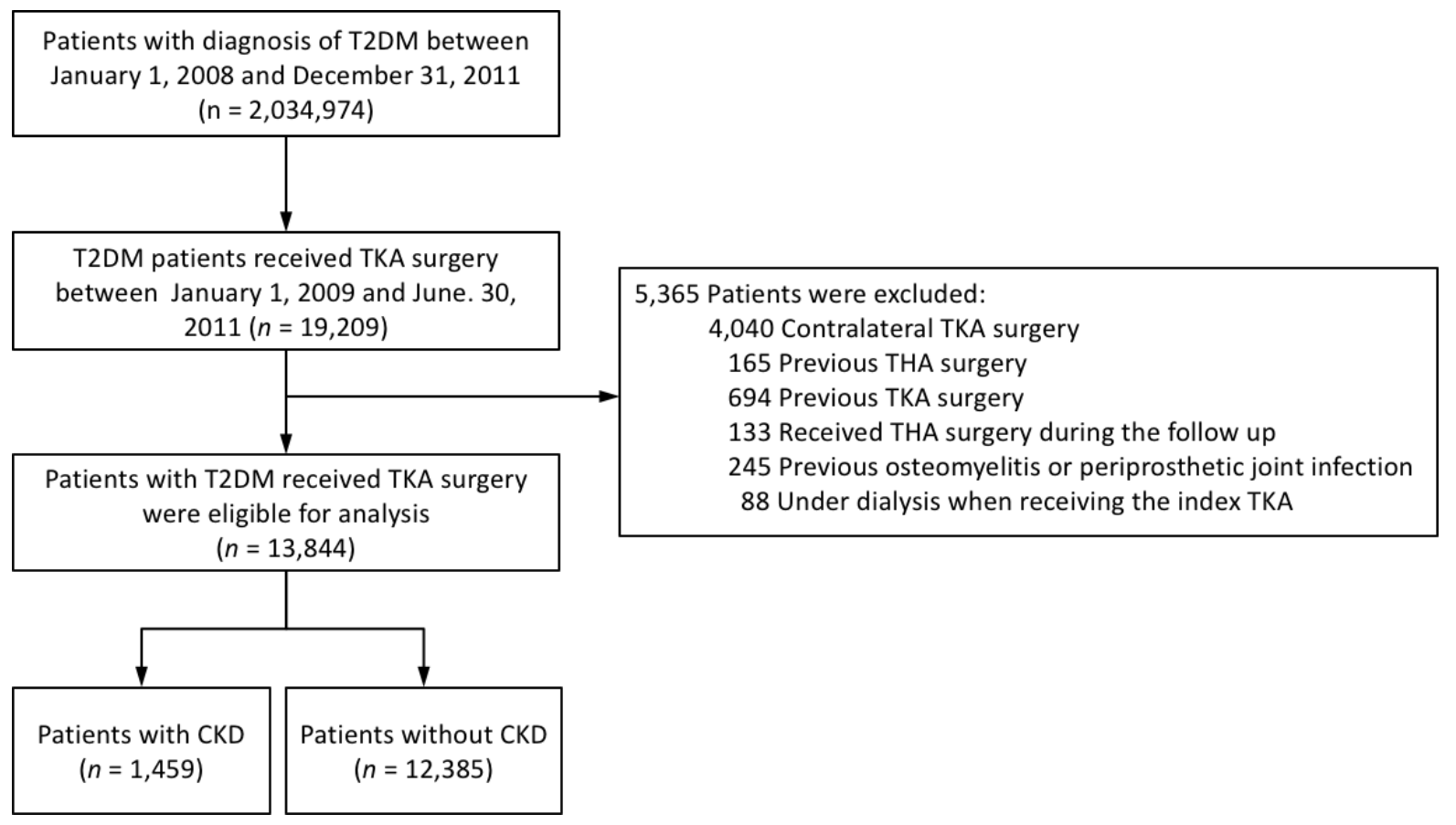

Figure 1: The enrollment of the study subjects. 
Table 1: Characteristics of the study cohort

\begin{tabular}{|c|c|c|c|}
\hline Variable & $\begin{array}{c}\text { CKD } \\
(n=1459)\end{array}$ & $\begin{array}{l}\text { Non-CKD } \\
(n=12385)\end{array}$ & $P$ value \\
\hline Age, Mean $(S D)$ & $71.6(7.6)$ & $70.3(7.6)$ & $<0.0001^{*}$ \\
\hline \multicolumn{4}{|l|}{ Age group } \\
\hline$<65$ years & $241(16.5)$ & $2466(19.9)$ & \multirow[t]{3}{*}{$<0.0001^{*}$} \\
\hline $65-74$ years & 718 (49.2) & $6386(51.6)$ & \\
\hline$\geq 75$ years & $500(34.3)$ & $3533(28.5)$ & \\
\hline \multicolumn{4}{|l|}{ Gender } \\
\hline Male & $477(32.7)$ & $2738(22.1)$ & $<0.0001^{*}$ \\
\hline Female & $982(67.3)$ & 9647 (77.9) & $<0.0001^{*}$ \\
\hline \multicolumn{4}{|l|}{ Comorbidity } \\
\hline Hypertension & $1305(89.4)$ & $10140(81.9)$ & $<0.0001^{*}$ \\
\hline Dyslipidemia & 611 (41.9) & $5159(41.7)$ & 0.8703 \\
\hline Gouty arthritis & $366(25.1)$ & $1262(10.2)$ & $<0.0001^{*}$ \\
\hline Cirrhosis & $31(2.1)$ & $165(1.3)$ & $0.0154^{*}$ \\
\hline Ischemic heart disease & $421(28.9)$ & $2740(22.1)$ & $<0.0001^{*}$ \\
\hline Rheumatoid arthritis & $50(3.4)$ & $380(3.1)$ & 0.4550 \\
\hline COPD & $128(8.8)$ & $685(5.5)$ & $<0.0001^{*}$ \\
\hline Immune disease & $67(4.6)$ & $466(3.8)$ & 0.1193 \\
\hline Malignancy & $99(6.8)$ & $552(4.5)$ & $<0.0001^{*}$ \\
\hline Pulmonary embolism & $5(0.3)$ & $4(0.0)$ & $<0.0001^{*}$ \\
\hline Deep vein thrombosis & $15(1.0)$ & $51(0.4)$ & $0.0012^{*}$ \\
\hline Cerebral vascular accident & $145(9.9)$ & $1046(8.5)$ & 0.0545 \\
\hline Follow up (years) & $1.9(1.0)$ & $2.1(1.0)$ & $<0.0001^{*}$ \\
\hline
\end{tabular}

$\mathrm{CKD}=$ chronic kidney disease $; \mathrm{COPD}=$ chronic obstructive pulmonary disease; $\mathrm{SD}=$ standard deviation.

${ }^{*} P$ value $<0.05$.

\section{Cumulative incidence of PJI}

Forty-three (3.0\%) and $283(2.3 \%)$ patients suffered from TKA infections during follow-up in the CKD and non-CKD groups, respectively. Among these 326 patients, it took 4.7 months to reach $50 \%$ cumulative incidence of superficial wound infection (PJI just requiring antibiotic treatment), and approximately 10 months to reach $50 \%$ cumulative incidence of infection requiring simple surgical debridement or implant removal (Figure 3).

We used propensity score matching method to compare the difference between CKD cohort and NonCKD cohort. We enrolled totally 2918 patients (1459 in each group). There was no significant difference in comorbidities between two groups (Supplementary Table 1) after matching. CKD group had a higher risk of urinary tract infection than control group after matching
(Supplementary Table 2). In outcomes after discharge, CKD group had a comparable risk of AMI and MACE as control group after matching. For mortality, CKD group still have a higher risk of overall mortality and a borderline risk of mortality in 90 days after discharge than control group ( $P=0.0949$, Supplementary Table 3$)$.

\section{DISCUSSION}

The main findings of this study are that the diabetic patients with CKD had a higher incidence of urinary tract infections in the index hospitalization for TKA compared to diabetic patients without CKD. In addition, the patients with CKD had higher risks of acute myocardial infarction, major adverse cardiac events, all-cause mortality, and readmission during follow-up compared to those without 
Table 2: In-hospital complications

\begin{tabular}{|c|c|c|c|c|}
\hline Variable & $\begin{array}{c}\text { CKD } \\
(n=1459)\end{array}$ & $\begin{array}{l}\text { Non-CKD } \\
(n=12385)\end{array}$ & $\begin{array}{c}\text { CKD vs. Non-CKD } \\
\text { OR }(95 \% \mathrm{CI})^{\mathrm{a}}\end{array}$ & $P$ value \\
\hline Pneumonia & $4(0.3)$ & $28(0.2)$ & $0.91(0.31-2.68)$ & 0.8670 \\
\hline Urinary tract infection & $54(3.7)$ & $310(2.5)$ & $1.61(1.19-2.17)$ & $0.0020^{*}$ \\
\hline Acute PJI & $5(0.3)$ & $60(0.5)$ & $0.73(0.29-1.83)$ & 0.4979 \\
\hline In hospital death & $2(0.1)$ & $7(0.1)$ & $1.53(0.30-7.74)$ & 0.6075 \\
\hline
\end{tabular}

$\mathrm{PJI}=$ periprosthetic joint infection; $\mathrm{OR}=$ odds ratio; $\mathrm{CI}=$ confidence interval.

a Variables listed in Table 1 (except for follow up year) were adjusted.

${ }^{*} P$ value $<0.05$.

Table 3: Outcomes after discharge of the index admission

\begin{tabular}{|c|c|c|c|c|}
\hline Variables & $\begin{array}{c}\text { CKD } \\
(n=1459)\end{array}$ & $\begin{array}{l}\text { Non-CKD } \\
(n=12385)\end{array}$ & $\begin{array}{c}\text { CKD vs. Non-CKD HR } \\
(95 \% \text { CI })^{b}\end{array}$ & $P$ value \\
\hline \multicolumn{5}{|l|}{ Infection outcome } \\
\hline Superficial wound infection & $14(1.0)$ & $93(0.8)$ & $1.27(0.71-2.26)$ & 0.4252 \\
\hline PJI requiring debridement & $30(2.1)$ & $210(1.7)$ & $1.18(0.77-1.80)$ & 0.4397 \\
\hline PJI requiring implants removal & $8(0.6)$ & $35(0.3)$ & $1.76(0.80-3.85)$ & 0.1601 \\
\hline Any PJI & $43(3.0)$ & $283(2.3)$ & $1.31(0.94-1.82)$ & 0.1133 \\
\hline \multicolumn{5}{|l|}{ Cardiac outcome } \\
\hline Pulmonary embolism & $2(0.1)$ & $34(0.3)$ & $0.43(0.10-1.80)$ & 0.2479 \\
\hline Deep vein thrombosis & $13(0.9)$ & $117(0.9)$ & $0.77(0.44-1.34)$ & 0.3540 \\
\hline Cerebral vascular accident & $67(4.6)$ & $447(3.6)$ & $1.13(0.87-1.47)$ & 0.3661 \\
\hline Acute myocardial infarction & $22(1.5)$ & $75(0.6)$ & $2.06(1.26-3.39)$ & $0.0043^{*}$ \\
\hline Major adverse cardiac event $\mathrm{t}^{\mathrm{a}}$ & $89(6.1)$ & $531(4.3)$ & $1.24(0.98-1.56)$ & 0.0678 \\
\hline \multicolumn{5}{|l|}{ Re-admission } \\
\hline In 90 days & $252(17.3)$ & $1,291(10.4)$ & $1.52(1.32-1.75)$ & $<0.0001^{*}$ \\
\hline At the last follow up & $814(55.8)$ & $5,507(44.5)$ & $1.39(1.28-1.50)$ & $<0.0001^{*}$ \\
\hline \multicolumn{5}{|l|}{ Mortality due to any cause } \\
\hline In 90 days & $15(1.0)$ & $37(0.3)$ & $2.55(1.35-4.80)$ & $0.0038^{*}$ \\
\hline At the last follow up & $110(7.5)$ & $439(3.5)$ & $1.99(1.59-2.48)$ & $<0.0001^{*}$ \\
\hline
\end{tabular}

$\mathrm{PJI}=$ periprosthetic joint infection; $\mathrm{HR}=$ hazard ratio; $\mathrm{CI}=$ confidence interval.

a Any one of the cardiac outcomes.

${ }^{\mathrm{b}}$ Variables listed in Table 1 (except for follow-up years) were adjusted.

${ }^{*} P$ value $<0.05$.

CKD. Furthermore, the patients with CKD had a trend of a higher risk of developing PJI than those without CKD. Therefore, orthopaedic surgeons should pay more attention to this group of patients.

The relationship between CKD and periprosthetic joint infections is controversial. CKD has been reported to an independent risk factor for PJI following elective hip and knee arthroplasty [5], and McCleery et al. [12] also reported that patients with CKD have a greater risk of developing early and late PJIs following TKA. In addition, Miric et al. [15] reported that patients with CKD have a higher incidence of superficial wound infections than those without CKD. Other studies have also shown that patients with CKD have a comparable risk of PJI to those without CKD regardless of the type of infection $[16,17]$. However, most studies have not considered the influence 


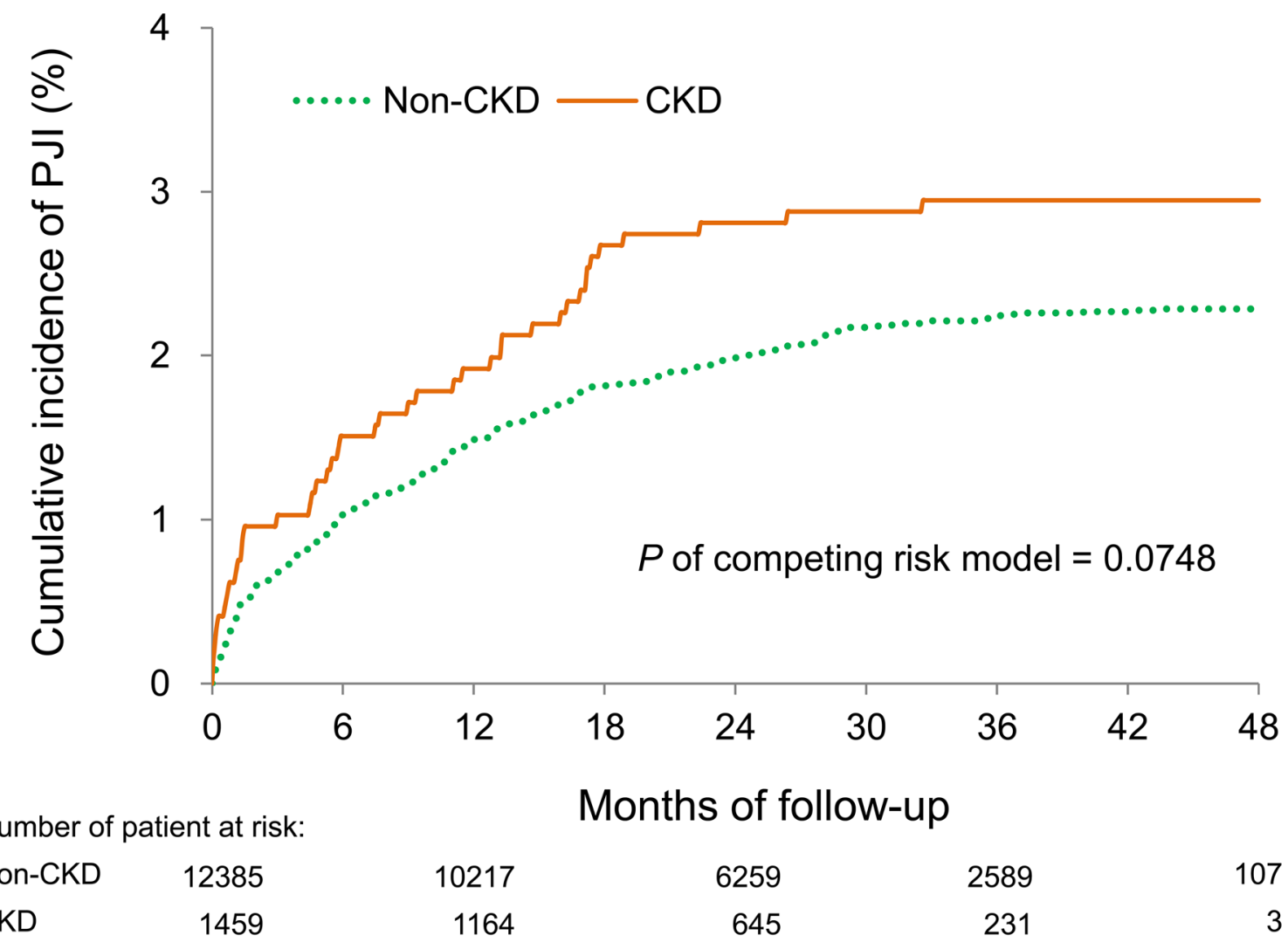

Figure 2: Cumulative incidence of PJI. Patients with CKD tended to have a higher cumulative incidence of PJI than those without $\mathrm{CKD}$, although the difference was not statistically significant $(P=0.0748$, competing risk survival model).

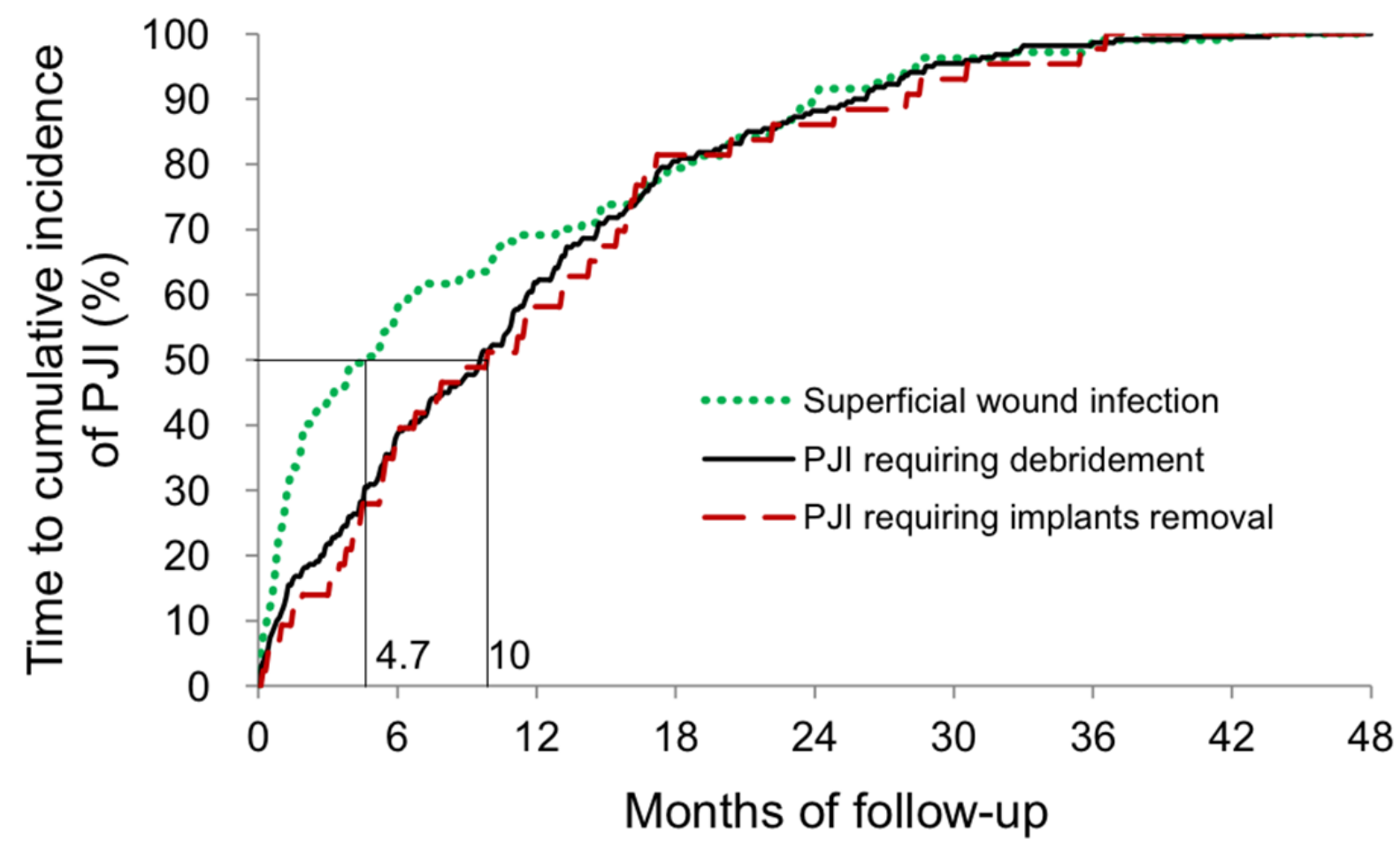

Figure 3: Time to the cumulative incidence of PJI. Superficial wound infections developing earlier than PJIs requiring surgical procedures (median time, 4.7 months versus 10 months). 
of DM, which may play a pivotal role in the associated outcomes. In this study, the patients with CKD had a trend of developing PJIs following TKA compared to those without CKD after adjusting for confounding factors including DM.

Most superficial wound infections develop earlier than deep joint infections requiring surgical debridement or removal of the implants following index surgery regardless of the type of PJI. This may be due to the sequential treatment protocol, and because implant removal is always the ultimate procedure to control an infection. Further studies focusing on outcomes including duration of infection silence and survival with implants are needed to elucidate the efficacy of each treatment protocol on PJI.

Several articles have reported that impaired renal function is an independent risk factor for early 90day mortality and 30-day morbidity after total joint arthroplasty [5, 9, 11, 17-20]. Ackland et al. [8] reported that patients with CKD had higher rates of pulmonary, infectious, and cardiovascular complications than those without CKD as well as a longer hospital stay by 4 days. In addition, Warth et al. [16] also demonstrated an increased risk of cardiac and systemic infectious complications as well as overall morbidity and mortality.

Mathew et al. [21] reported that patients with CKD had a higher incidence of postoperative death and cardiac vascular events than those without CKD during follow-up after non-cardiac surgery. Warth et al. [16] also reported that patients with CKD had a higher incidence of cardiac events, urinary tract infections and mortality than those without CKD after TKA, although the confounding factor of DM was not well evaluated since the CKD group had a significantly higher incidence of DM than the nonCKD group. The present study further demonstrates that patients with CKD have a higher risk of cardiac events and all-cause mortality after adjustments for all possible contributing confounding factors including DM.

\section{Strengths and limitations}

There are three limitations to this study. First, although this study is a national health database study, it has it's a natural retrospective design and relatively small number of patients. Second, we used ICD-9-CM codes to identify the diagnosis of CKD, and could not further divide the patients according to the severity of disease or clinical data. However, in Taiwan, the diagnosis of CKD is only attributed to a substantial deterioration in renal function, and therefore we can assume that these patients had at least stage III CKD. Further study to explore the associations between different severity of CKD and TKA outcome is required. Third, we used ICD-9-CM codes to identify the outcomes following TKA, and some minor adverse outcomes not requiring admission may have been missed. However, the critical identification of events ensured that the actual event rate could only have been more severe than our estimation, not lower.

There are also several strengths to this study. First, in contrast to previous studies, we stratified the effect of DM and further consolidated the effect of CKD itself on the surgical outcomes of TKA. Second, we not only used ICD-9-CM diagnostic codes to define PJI, but also validated the diagnoses using surgical codes in the NHIRD. This strict definition helped to strengthen the investigation of the relationship between the CKD and PJI.

In conclusion, compared to the diabetic patients without $\mathrm{CKD}$, those with $\mathrm{CKD}$ had a higher incidence of urinary tract infection in the index admission, and higher risks of acute myocardial infarction, major adverse cardiac events, all-cause mortality, and readmission during followup. The patients with CKD also had a trend of a higher risk developing PJIs than those without CKD. To improve outcomes, orthopaedic surgeons should pay more attention to these patients.

\section{MATERIALS AND METHODS}

\section{Data source}

Data were obtained from the National Health Insurance Research Database (NHIRD) of Taiwan. This single-payer National Health Insurance (NHI) program was launched in 1995, and currently more than 23 million people (almost 99\% of the population) are enrolled. The NHIRD contains claims data submitted by medical institutions through the NHI program. All clinical diagnoses are registered using International Classification of Diseases, 9th Revision, Clinical Modification [ICD9-CM] codes (http://www.icd9-data.com/2007). The accuracy of the diagnoses of major diseases in the NHIRD such as DM or CKD has previously been validated [2224]. All patient data are de-identified prior to analysis to ensure anonymity. This study was reviewed and approved by the Ethics Institutional Review Board of Chang Gung Memorial Hospital (IRB CGMH 103-5040B).

\section{Study cohort enrollment and exclusion criteria}

All patients in the NHIRD with type 2 DM (ICD9-CM code 250) were identified from January 1, 2008 to December 31, 2011 according to the method previously described [15]. Only patients with type $2 \mathrm{DM}$ who were hospitalized for TKA between January 1, 2009 and June 30, 2011 were included in this study to ensure at least 1 year of previous medical records to identify comorbidities and exclusion conditions and to make sure each patient was followed for at least 6 months (Figure 1). The index hospitalization was defined as the admission for which the patient was admitted for TKA. CKD was defined according to the ICD-9-CM diagnostic codes detailed in Supplementary Table 1 [25]. The follow-up period was 
recorded from the index hospitalization to the date of death or until December 31, 2011.

In order to enroll patients with a first-ever procedure, the patients who had a history of TKA or total hip arthroplasty (THA) surgery were excluded. Patients with other factors that could confound the outcome were also excluded, including those with a history of osteomyelitis. Immunocompromised patients such as those undergoing dialysis were also excluded. Finally, 13844 patients were enrolled in the study cohort. These patients were then classified into two groups according to whether or not they had CKD. The study subject enrollment process is shown in Figure 1.

\section{Comorbidities and outcomes}

Baseline comorbidities were identified by ICD-9$\mathrm{CM}$ diagnostic codes before the index hospitalization. Chronic diseases such as DM and hypertension were defined as repeated diagnoses in separate clinic visits 1 year before the index admission. Acute comorbidities and events such as pulmonary embolism were defined as one inpatient primary diagnosis during a prior hospitalization.

In-hospital complications including PJI (i.e. superficial wound infection, surgical debridement, and implant removal), pneumonia, urinary tract infection, and death during the index admission were recorded. Infection outcomes were defined as composite outcomes of superficial wound infection, PJI requiring debridement, and PJI requiring implant removal during the followup period. A superficial wound infection was defined as readmission due to an infection only requiring antibiotic treatment. A PJI requiring debridement was defined as surgical debridement, arthrotomy, or capsulectomy requiring hospitalization. Cardiac outcomes included hospitalization due to pulmonary embolism, deep vein thrombosis, cerebral vascular event and acute myocardial infarction. A major adverse cardiac event was defined as any of the cardiac outcomes (Appendix 1). Deathwas identified by the method described in the previous study from the NHIRD in Taiwan [26].

\section{Statistical analysis}

Baseline characteristics between the CKD and nonCKD groups were compared using the t-test for continuous variables and chi-square test for categorical variables. The incidence of in-hospital complications between the two groups was compared using multivariate logistic regression analysis with adjustments for all potential confounders listed in Table 1 except for follow-up years. With regards to the late complication after discharge of the index admission, we performed subdistribution hazard model in which death during the follow up was considered as a competing risk to compare group differences in which the aforementioned confounders were also adjusted for [27]. The cumulative incidence of long-term PJIs was estimated using the Kaplan-Meier method, and the log-rank test was used to compare group differences. All analyses were performed using SAS statistical software (version 9.4; SAS Institute Inc., Cary, NC). A $P$ value $<0.05$ was considered to be statistically significant.

\section{Abbreviations}

$\mathrm{CI}$, confidence interval; CKD, chronic kidney disease; COPD, chronic obstructive pulmonary disease; DM, diabetes mellitus; HR, hazard ratio; ICD-9-CM, International Classification of Diseases, 9th Revision, Clinical Modification; NHI, National Health Insurance; NHIRD, National Health Insurance Research Database; OR, odds ratio; PJI, periprosthetic joint infection; SD, standard deviation; TKA, total knee arthroplasty; THA, total hip arthroplasty.

\section{Author contributions}

Liang-Tseng Kuo, Su-Ju Lin and Tien-Hsing Chen designed the study.

Tien-Hsing Chen and Liang-Tseng Kuo conducted the data mining and statistics.

Chi-Lung Chen and Pei-An Yu prepared the tables and figures.

Liang-Tseng Kuo, Wei-Hsiu Hsu, and Tien-Hsing

Chen wrote the main manuscript.

All authors reviewed the manuscript.

\section{CONFLICTS OF INTEREST}

The authors declare no conflicts of interest.

\section{FUNDING}

None.

\section{REFERENCES}

1. Maier GS, Horas K, Seeger JB, Roth KE, Kurth AA, Maus U. Is there an association between periprosthetic joint infection and low vitamin D levels? Int Orthop. 2014;38:1499-504.

2. Baek SH. Identification and preoperative optimization of risk factors to prevent periprosthetic joint infection. World J Orthop. 2014;5:362-7.

3. George DA, Drago L, Scarponi S, Gallazzi E, Haddad FS, Romano CL. Predicting lower limb periprosthetic joint infections: a review of risk factors and their classification. World J Orthop. 2017;8:400-1.

4. Phillips JE, Crane TP, Noy M, Elliott TS, Grimer RJ. The incidence of deep prosthetic infections in a specialist orthopaedic hospital: a 15-year prospective survey. J Bone Joint Surg Br. 2006;88:943-8. 
5. Bozic KJ, Lau E, Kurtz S, Ong K, Rubash H, Vail TP, Berry DJ. Patient-related risk factors for periprosthetic joint infection and postoperative mortality following total hip arthroplasty in Medicare patients. J Bone Joint Surg Am. 2012;94:794-800.

6. Serna F, Mont MA, Krackow KA, Hungerford DS. Total knee arthroplasty in diabetic patients. Comparison to a matched control group. J Arthroplasty. 1994;9:375-9.

7. Bolognesi MP, Marchant MH Jr, Viens NA, Cook C, Pietrobon R, Vail TP. The impact of diabetes on perioperative patient outcomes after total hip and total knee arthroplasty in the United States. J Arthroplasty. 2008;23:92-8

8. Ackland GL, Moran N, Cone S, Grocott MP, Mythen MG. Chronic kidney disease and postoperative morbidity after elective orthopedic surgery. Anesth Analg. 2011;112:1375-81.

9. Belmont PJ Jr, Goodman GP, Hamilton W, Waterman BR, Bader JO, Schoenfeld AJ. Morbidity and mortality in the thirty-day period following total hip arthroplasty: risk factors and incidence. J Arthroplasty. 2014;29:2025-30.

10. Kuo LT, Lin SJ, Hsu WH, Peng KT, Lin CL, Hsu RW. The effect of renal function on surgical outcomes of intracapsular hip fractures with osteosynthesis. Arch Orthop Trauma Surg. 2014;134:39-45.

11. Kuo FC, Lin PC, Lu YD, Lee MS, Wang JW. Chronic kidney disease is an independent risk factor for transfusion, cardiovascular complication, and thirty-day readmission in minimally invasive total knee arthroplasty. J Arthroplasty. 2017;32:1630-4.

12. Cavanaugh PK, Chen AF, Rasouli MR, Post ZD, Orozco FR, Ong AC. Complications and mortality in chronic renal failure patients undergoing total joint arthroplasty: a comparison between dialysis and renal transplant patients. J Arthroplasty. 2016;31:465-72.

13. Sunday JM, Guille JT, Torg JS. Complications of joint arthroplasty in patients with end-stage renal disease on hemodialysis. Clin Orthop Relat Res. 2002;397:350-5.

14. McCleery MA, Leach WJ, Norwood T. Rates of infection and revision in patients with renal disease undergoing total knee replacement in Scotland. J Bone Joint Surg Br. 2010;92:1535-9.

15. Miric A, Inacio MC, Namba RS. Can total knee arthroplasty be safely performed in patients with chronic renal disease? Acta Orthop. 2014;85:71-8.

16. Warth LC, Pugely AJ, Martin CT, Gao Y, Callaghan JJ. Total joint arthroplasty in patients with chronic renal disease: is it worth the risk? J Arthroplasty. 2015;30:51-4.
17. Deegan BF, Richard RD, Bowen TR, Perkins RM, Graham JH, Foltzer MA. Impact of chronic kidney disease stage on lower extremity arthroplasty. Orthopedics. 2014;37:e613-8.

18. Jämsen E, Puolakka T, Eskelinen A, Jäntti P, Kalliovalkama J, Nieminen J, Valvanne J. Predictors of mortality following primary hip and knee replacement in the aged. A singlecenter analysis of 1,998 primary hip and knee replacements for primary osteoarthritis. Acta Orthop. 2013;84:44-53.

19. Memtsoudis SG, Della Valle AG, Besculides MC, Esposito M, Koulouvaris P, Salvati EA. Risk factors for perioperative mortality after lower extremity arthroplasty: a populationbased study of 6,901,324 patient discharges. J Arthroplasty. 2010;25:19-26.

20. Pugely AJ, Callaghan JJ, Martin CT, Cram P, Gao Y. Incidence of and risk factors for 30-day read- mission following elective primary total joint arthroplasty: analysis from the ACS- NSQIP. J Arthroplasty. 2013;28:1499-504.

21. Mathew A, Devereaux PJ, O'Hare A, Tonelli M, ThiessenPhilbrook H, Nevis IF, Iansavichus AV, Garg AX. Chronic kidney disease and postoperative mortality: a systematic review and meta-analysis. Kidney Int. 2008;73:1069-81.

22. Lin CC, Lai MS, Syu CY, Chang SC, Tseng FY. Accuracy of diabetes diagnosis in health insurance claims data in Taiwan. J Formos Med Assoc. 2005;104:157-63.

23. Cheng CL, Kao YH, Lin SJ, Lee CH, Lai ML. Validation of the National Health Insurance Research Database with ischemic stroke cases in Taiwan. Pharmacoepidemiol Drug Saf. 2011;20:236-42.

24. Chang CH, Lee YC, Tsai CT, Chang SN, Chung YH, Lin MS, Lin JW, Lai MS. Continuation of statin therapy and a decreased risk of atrial brillation/utter in patients with and without chronic kidney disease. Atherosclerosis. 2014;232:224-30.

25. Lee YC, Hung SY, Wang HK, Lin CW, Wang HH, Chen SW, Chang MY, Ho LC, Chen YT, Liou HH, Tsai TC, Tseng SH, Wang WM, et al. Sleep apnea and the risk of chronic kidney disease: a nationwide population-based cohort study. Sleep. 2015;38:213-21.

26. Wu CY, Chen YJ, Ho HJ, Hsu YC, Kuo KN, Wu MS, Lin JT. Association between nucleoside analogues and risk of hepatitis $\mathrm{B}$ virus-related hepatocellular carcinoma recurrence following liver resection. JAMA. 2012;308:1906-14.

27. Fine JP, Gray RJ. A proportional hazards model for the subdistribution of a competing risk. J Am Stat Assoc. 1999;94:496-509. 